\title{
Repairing nanoscale scratched grooves on polycrystalline ceramics using optical near-field assisted sputtering
}

\author{
W. Nomura $\cdot$ T. Yatsui $\cdot$ Y. Yanase $\cdot$ K. Suzuki $\cdot$ \\ M. Fujita $\cdot$ A. Kamata $\cdot$ M. Naruse $\cdot$ M. Ohtsu
}

Received: 2 October 2009 / Published online: 1 November 2009

(C) The Author(s) 2009. This article is published with open access at Springerlink.com

\begin{abstract}
We propose an optical near-field assisted sputtering method for repairing scratches on the surface of polycrystalline ceramics in a self-assembling manner. $\mathrm{An}^{\mathrm{Al}_{2} \mathrm{O}_{3}}$ source was sputtered on substrates with laser radiation of wavelength $473 \mathrm{~nm}$. The average depth of the scratched grooves on polycrystalline $\mathrm{Al}_{2} \mathrm{O}_{3}$ ceramic substrate decreased from $3.2 \mathrm{~nm}$ to $0.79 \mathrm{~nm}$. Using a Hough transform, we also confirmed the selective repair of scratches.
\end{abstract}

PACS 81.16.Mk $\cdot$ 81.15.Cd

\section{Introduction}

Recently, optical transmission loss in polycrystalline ceramics has been dramatically decreased to levels as low as those in single crystals. As a result, these transparent ceramics are attracting interest for applications in optical technology [14] for use as gain media for solid state lasers or optical windows [5-7]. To realize larger lasing efficiency or to reduce

W. Nomura $(\bowtie) \cdot$ T. Yatsui $\cdot$ M. Naruse $\cdot$ M. Ohtsu

School of Engineering, The University of Tokyo, 2-11-16, Yayoi, Bunkyo-ku, Tokyo 113-8656, Japan

e-mail: nomura@nanophotonics.t.u-tokyo.ac.jp

W. Nomura $\cdot$ T. Yatsui $\cdot$ M. Naruse $\cdot$ M. Ohtsu

The Nanophotonics Research Center, The University of Tokyo,

2-11-16, Yayoi, Bunkyo-ku, Tokyo 113-8656, Japan

Y. Yanase · K. Suzuki · M. Fujita - A. Kamata

Core Technology Center, Covalent Materials Corporation, 30,

Soya, Hadano-shi, Kanagawa, 257-8566, Japan

M. Naruse

National Institute of Information and Communications

Technology, 4-2-1 Nukui-kita, Koganei, Tokyo 184-8795, Japan the scattering loss in optical windows, further decreases in the surface roughness are required. To meet this requirement, chemical-mechanical polishing (CMP) has been employed on single crystalline and amorphous optical materials [8]. However, this method is difficult to apply to polycrystalline ceramics because of their anisotropic interaction with the polishing medium. A key problem with CMP is that it may cause scratches because of collisions with the abrasive grains in the slurry. Furthermore, the CMP may cause bumps on the surface, due to the difference in etching rates between adjacent grains in the polycrystal.

To solve these problems, we propose a novel method that makes use of the optical near field. Its outstanding advantage is not only its high spatial resolution, which is beyond the diffraction limit, but also its self-assembling reaction capability. Because localized optical near fields are generated preferably on a surface with nanoscale curvature, they can be induced on fine scratches, contributing to repair them. In addition, the repairing process stops automatically after the scratches disappear. Such selective self-assembling optical near-field interactions previously have been used for depositing nanoparticles, sputtering, and photochemical etching [9-11]. Here, we employ this novel technique to repair the scratches on polycrystalline $\mathrm{Al}_{2} \mathrm{O}_{3}$ ceramics.

\section{Experimental technique}

Figure 1 shows a schematic of our method. As a result of preliminary polish of the $\mathrm{Al}_{2} \mathrm{O}_{3}$ ceramic, its surface contains nanoscale scratched grooves. Because the edges of the grooves have larger surfaces areas than the flat surface, the sputtered $\mathrm{Al}_{2} \mathrm{O}_{3}$ particles have a higher deposition rate at the edge after migration on the surface $[12,13]$. As shown in Fig. 1(a), it therefore is expected that $\mathrm{Al}_{2} \mathrm{O}_{3}$ will be 
deposited preferentially at the edges of scratched grooves, which will not help to repair the scratches. To avoid extra deposition on the edge of the grooves and to repair the scratches, we used optical near-field desorption [10]. Because the optical near field (i.e., the dressed photon) can ex-
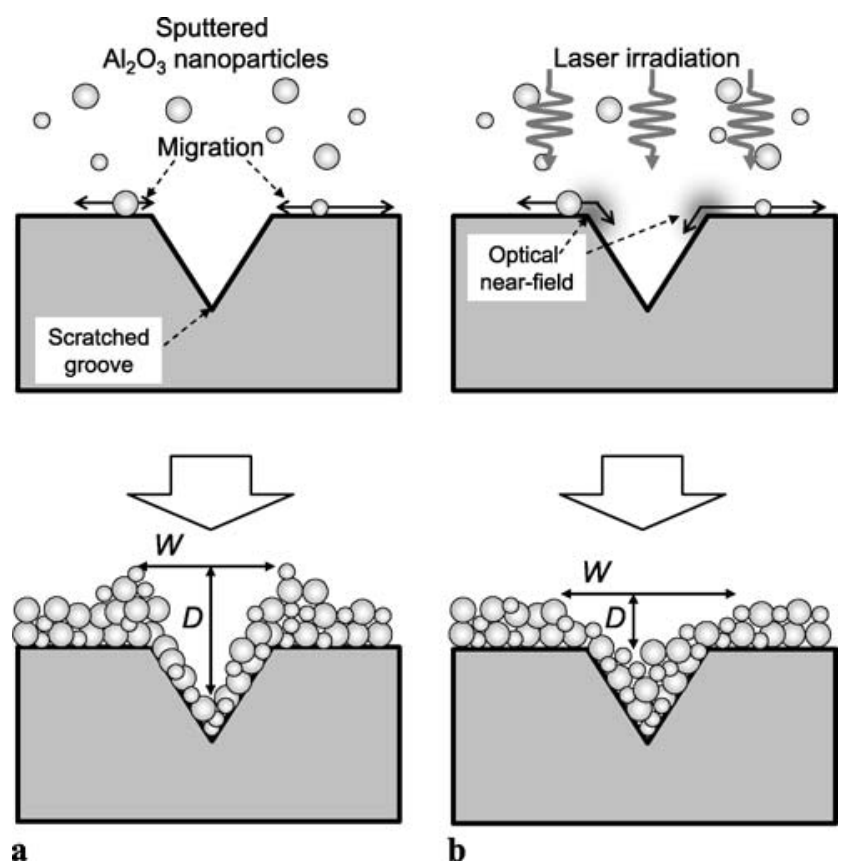

Fig. 1 Schematics of deposition on the scratched substrate surface (a) without and (b) with irradiation during the sputtering. $D$ is the depth and $W$ is the width of the scratched groove cite coherent phonons in the nanoscale structure, a virtual exciton-phonon-polariton (EPP) is generated on the substrate. A multistep transition via the EPP can accelerate the photochemical reaction although the photon energy is lower than the absorption band edge energy of the material [14]. When the ceramic is irradiated, a highly localized optical near field is generated at the edges of scratches, causing the photodesorption of depositing $\mathrm{Al}_{2} \mathrm{O}_{3}$ nanoparticles. As shown in Fig. 1(b), if the light has a lower energy than the absorption band edge of the nanoparticles, effective deposition will decrease at the edge, and $\mathrm{Al}_{2} \mathrm{O}_{3}$ will accumulate on the bottom of the groove [10]. This process automatically stops after the scratches disappear, so that the optical near field can no longer be generated.

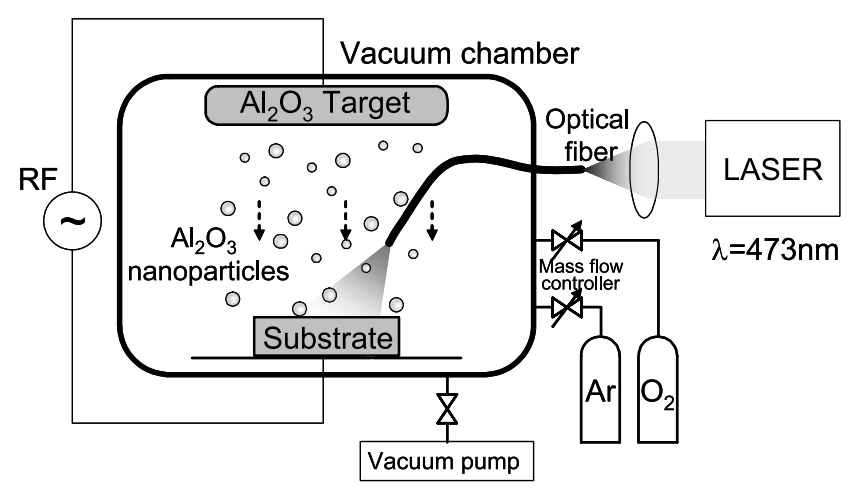

Fig. 2 Schematic of the experimental setup
Fig. 3 AFM images of substrates (a) before sputtering (substrate $A$ ), (b) after sputtering without irradiation (substrate $B$ ), and (c) after sputtering with irradiation (substrate $C)$. (d), (e), and (f) Cross-sectional profiles along the white dashed lines in (a), (b), and (c), respectively
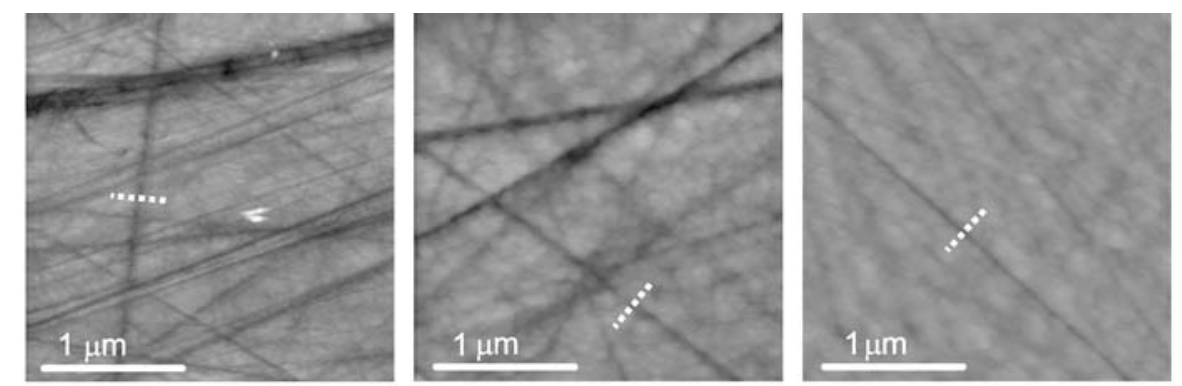

$\mathbf{a}$

b

c
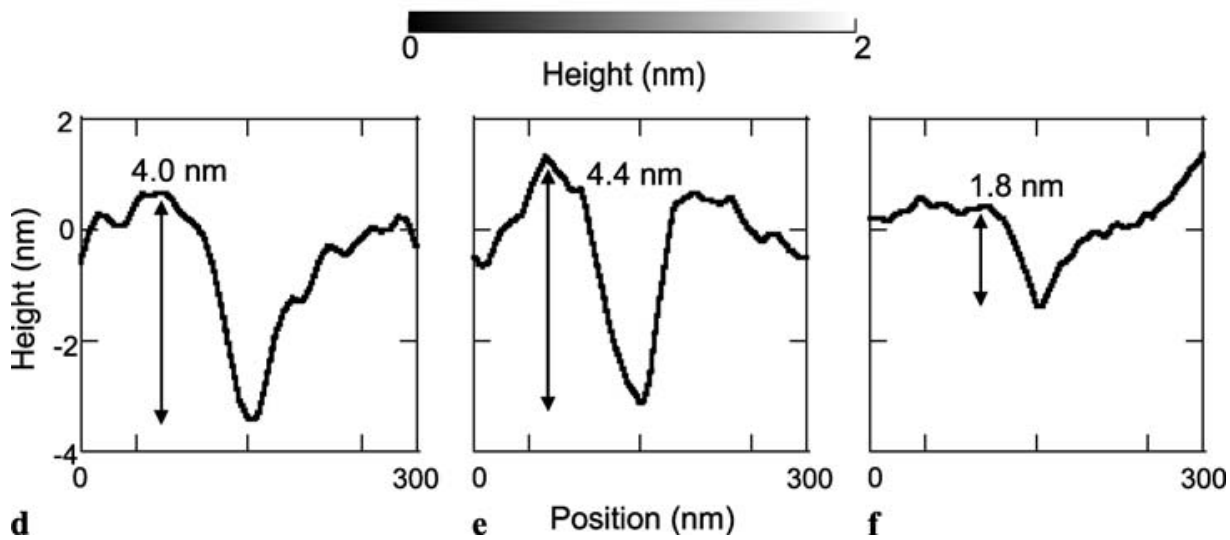
We performed optical near-field assisted sputtering to repair the scratches on the surface of the translucent $\mathrm{Al}_{2} \mathrm{O}_{3}$ ceramic SAPPHAL ${ }^{\circledR}{ }^{1}$ Figure 2 shows a schematic of the experimental setup. Planar surfaces of SAPPHAL ${ }^{\circledR}$ substrate were prepared by polishing using diamond abrasive grains with a diameter of $0.5 \mu \mathrm{m}$. The $\mathrm{Al}_{2} \mathrm{O}_{3}$ was deposited using radio-frequency $(\mathrm{RF})$ magnetron sputtering (RF power: $300 \mathrm{~W}$; frequency: $13 \mathrm{MHz}$ ). The total gas pressure was $7 \times 10^{-1} \mathrm{~Pa}$, with a gas flow of $16 \mathrm{sccm} \mathrm{Ar}$ and $1.2 \mathrm{sccm}$ $\mathrm{O}_{2}$ [15]. We also used SAPPHAL ${ }^{\circledR}$ as the target material for sputtering. The $\mathrm{CW}$ second harmonic of a Nd:YAG laser with a wavelength of $\lambda=473 \mathrm{~nm}$ was used as the light source for optical near-field generation. During the sputtering process, the surface was irradiated with light with an optical power density of $2.7 \mathrm{~W} \mathrm{~cm}^{-2}$. As stated previously, the photon energy of this laser was lower than the absorption band edge energy of $\mathrm{Al}_{2} \mathrm{O}_{3}(\lambda=250 \mathrm{~nm})$ [16]. The light was introduced to the ceramic surface through a multimode optical fiber. After $30 \mathrm{~min}$ of sputtering, the thickness of the deposited $\mathrm{Al}_{2} \mathrm{O}_{3}$ layer was about $100 \mathrm{~nm}$. We observed the surface of the substrates using an atomic force microscope (AFM).

\section{Results and discussion}

Figures 3(a), (b), and (c) show typical AFM images of SAPPHAL ${ }^{\circledR}$ surfaces before sputtering, after sputtering without irradiation, and after sputtering with irradiation, respectively. Figures 3(d), (e), and (f) show the cross-sectional profiles of typical scratches along the white dashed lines in Figs. 3(a), (b), and (c), respectively. The depth of the scratched grooves in Fig. 3(e) is $4.4 \mathrm{~nm}$, which is deeper than that in Fig. 3(d) $(4.0 \mathrm{~nm})$. This was caused by the extra deposition at the edges of the scratch due to the low surface potential, as described in Fig. 1(a). In contrast, Fig. 3(f) shows that the depth decreased to $1.8 \mathrm{~nm}$ without extra deposition at the edges of scratches after sputtering with irradiation. For more quantitative evaluation, we calculated the surface roughness $R a$. The $R_{a}$ values over the AFM images of Figs. 3(a)-(c) were $R a_{A}=1.3 \mathrm{~nm}, R a_{B}=1.1 \mathrm{~nm}$, and $R a_{C}=0.49 \mathrm{~nm}$, respectively. These results indicated that the repair of scratched grooves by optical near-field desorption at the edge of the scratches resulted in a drastic decrease in the surface roughness.

To selectively evaluate the profiles of the scratched grooves, we used the Hough transform [17]. After the AFM image of Fig. 4(a) was leveled by a least squares-method and binarized, the linear features of scratches were automatically extracted using a Hough transform; see Fig. 4(b).

\footnotetext{
${ }^{1} \mathrm{~A}$ product of the Covalent Materials Corporation: http://www. covalent.co.jp/.
}
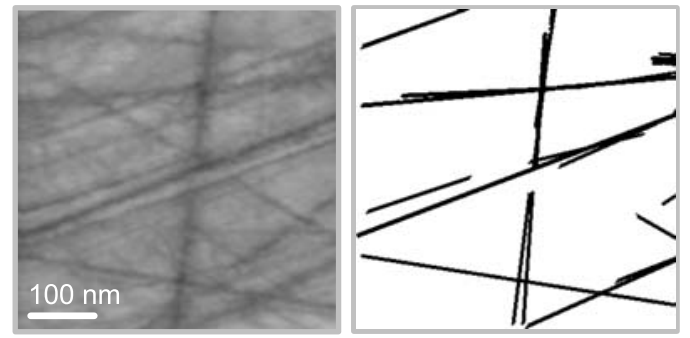

a

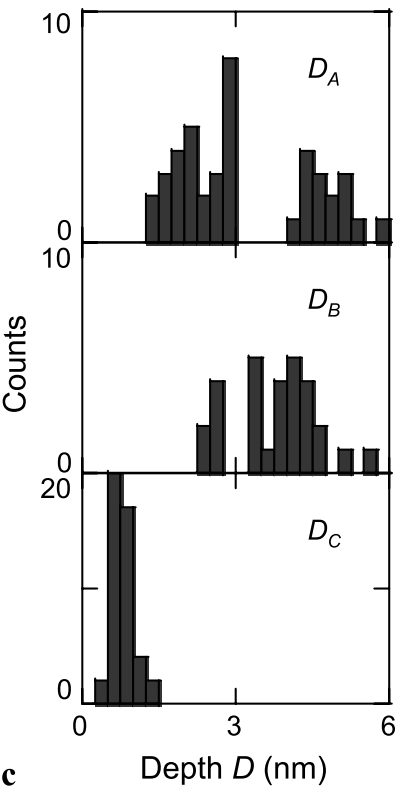

b

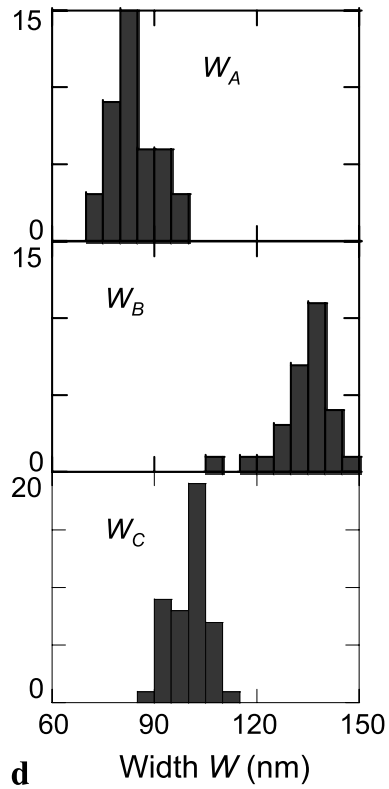

d

Width $W(\mathrm{~nm})$

Fig. 4 (a) An AFM image is leveled by a least-squares method. (b) Schematic of the Hough transform. Straight lines of the scratches are detected using the Hough transform of (a). (c) Histograms of depths, $D_{n}$. (d) Widths $\left(W_{n}\right)$ of scratches on alumina ceramics substrates; $A$ (before sputtering), $B$ (after sputtering without irradiation), and $C$ (after sputtering with irradiation)

Through this method we obtained the depth $D$ and width $W$ of the detected scratches. Figures 4(c) and (d) show statistical analyses of $D$ and $W$ obtained from the images in Figs. 3(a), (b), and (c), respectively. As shown in Fig. 4(c), the average values of $D$ were $\overline{D_{A}}=3.2 \mathrm{~nm}, \overline{D_{B}}=3.8 \mathrm{~nm}$, and $\overline{D_{C}}=0.79 \mathrm{~nm}$, respectively, which confirmed that the depth of the scratches was drastically decreased using nearfield assisted sputtering. Figure 4(d) shows that the width of the scratches after the sputtering without irradiation $\left(W_{B}\right)$ was increased beyond the original value $W_{A}$, which also supports our deposition model; see Fig. 1(a). In addition, the width $W_{C}$ also increased in comparison to $W_{A}$, supporting the model of Fig. 1(b). Further decreases in the width could be achieved by optimizing laser and sputtering conditions.

\section{Conclusion}

In conclusion, we proposed an optical near-field assisted sputtering method for repairing the scratched grooves on a 
translucent polycrystalline $\mathrm{Al}_{2} \mathrm{O}_{3}$ ceramic surface. We confirmed that the depth of the grooves was decreased by RF sputtering of $\mathrm{Al}_{2} \mathrm{O}_{3}$ with laser irradiation at a wavelength of $\lambda=473 \mathrm{~nm}$. We found a drastic decrease in surface roughness and obtained an average depth of $0.79 \mathrm{~nm}$. Furthermore, we analyzed the surface profile using a Hough transform. This approach clearly revealed the effects of the optical near-field assisted sputtering, which selectively repaired the scratched grooves. We believe our technique is applicable to a variety of substrates, including ceramic and crystal substrates. Furthermore, this method is compatible with mass production.

Acknowledgement This work was supported by the New Energy and Industrial Technology Development Organization (NEDO) Special Courses: A comprehensive activity for personnel training and industryacademia collaboration based on NEDO projects, and NEDO under the Research and Development Program of Innovative Energy Efficiency Technology.

Open Access This article is distributed under the terms of the Creative Commons Attribution Noncommercial License which permits any noncommercial use, distribution, and reproduction in any medium, provided the original author(s) and source are credited.

\section{References}

1. A. Ikesue, I. Furusato, J. Am. Ceram. Soc. 78, 225 (1995)

2. A. Ikesue, T. Kinoshita, K. Kmata, K. Yoshida, J. Am. Ceram. Soc. 78, 1033 (1995)

3. J. Lu, J. Son, M. Prabhu, J. Xu, K. Ueda, H. Yagi, T. Yanagitani, A. Kudryashov, Jpn. J. Appl. Phys. 39, L1048 (2000)

4. N. Tanaka, Bull. Ceram. Soc. Jpn. 38, 967 (2003)

5. A. Krell, P. Blank, H. Ma, T. Hutzler, J. Am. Ceram. Soc. 86, 12 (2003)

6. J. Lu, K. Ueda, H. Yagi, T. Yanagitani, Y. Akiyama, A.A. Kaminskii, J. Alloys Compd. 341, 220 (2002)

7. A. Ikesue, Opt. Mater. 19, 183 (2002)

8. L.M. Cook, J. Non-Cryst. Solids 120, 152 (1990)

9. T. Yatsui, W. Nomura, M. Ohtsu, IEICE Trans. Electron. E88-C, 1798 (2005)

10. T. Yatsui, W. Nomura, M. Ohtsu, Nano Lett. 5, 2548 (2005)

11. T. Yatsui, K. Hirata, W. Nomura, Y. Tabata, M. Ohtsu, Appl. Phys. B 93, 55 (2008)

12. F.J. Himpsel, J.E. Ortega, G.J. Mankey, R.F. Willis, Adv. Phys. 47, 511 (1998)

13. E.J. Menke, Q. Li, R.M. Penner, Nano Lett. 4, 2009 (2004)

14. T. Kawazoe, K. Kobayashi, S. Takubo, M. Ohtsu, J. Chem. Phys. 122, 024715 (2005)

15. J.B. Wachtman, A.R. Haber, Ceramic Films and Coatings (William Andrew Publishing/Noyes, New York/Norwich, 1993)

16. F. Benabid, M. Notcutt, V. Loriette, L. Ju, D.G. Blair, J. Phys. D 33, 589 (2000)

17. R.O. Duda, P.E. Hart, Commun. ACM 15, 11 (1972) 